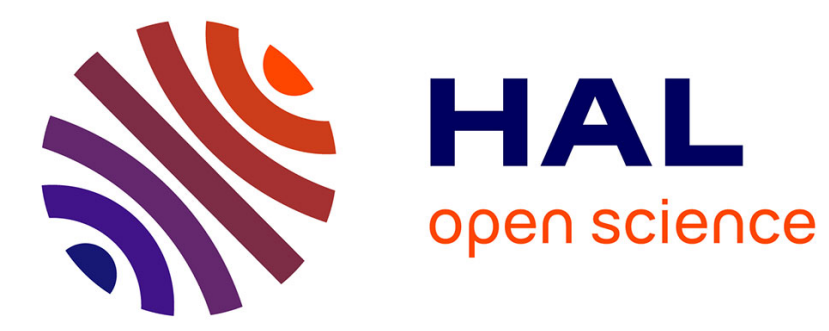

\title{
Investigating reaction pathways in rare events simulations of antibiotics diffusion through protein channels
}

Eric Hajjar, Amit Kumar, Paolo Ruggerone, Matteo Ceccarelli

\section{- To cite this version:}

Eric Hajjar, Amit Kumar, Paolo Ruggerone, Matteo Ceccarelli. Investigating reaction pathways in rare events simulations of antibiotics diffusion through protein channels. Journal of Molecular Modeling, 2010, 16 (11), pp.1701-1708. 10.1007/s00894-010-0698-4 . hal-00586136

\section{HAL Id: hal-00586136 \\ https://hal.science/hal-00586136}

Submitted on 15 Apr 2011

HAL is a multi-disciplinary open access archive for the deposit and dissemination of scientific research documents, whether they are published or not. The documents may come from teaching and research institutions in France or abroad, or from public or private research centers.
L'archive ouverte pluridisciplinaire HAL, est destinée au dépôt et à la diffusion de documents scientifiques de niveau recherche, publiés ou non, émanant des établissements d'enseignement et de recherche français ou étrangers, des laboratoires publics ou privés. 


\section{Editorial Manager(tm) for Journal of Molecular Modeling Manuscript Draft}

Manuscript Number: JMM01132R2

Title: Investigating reaction pathways in rare events simulations of antibiotics diffusion through protein channels

Article Type: Special Issue Prague

Keywords: Reaction coordinate; free energy; accelerated molecular dynamics; antibiotics uptake; diffusion; bacterial porins; antibacterial design; ampicillin; moxifloxacine

Corresponding Author: Dr. Eric Hajjar,

Corresponding Author's Institution: University of Cagliari

First Author: eric hajjar, PhD

Order of Authors: eric hajjar, PhD; Eric Hajjar; amit kumar, JD; paolo ruggerone, PhD; matteo ceccarelli, $\mathrm{PhD}$

Manuscript Region of Origin:

Abstract: In Gram-negative bacteria, outer-membrane protein channels, such as OmpF of Escherichia coli, constitute the entry point of various classes of antibiotics. While antibacterial research and development is declining, bacterial resistance to antibiotics is rising and there is an emergency call for a new way to develop potent antibacterial agents and to bring them to the market faster and at reduced cost. An emerging strategy is to follow a bottom-up approach based on microscopically founded computational based screening, however such strategy needs better-tuned methods. Here we propose to use molecular dynamics (MD) simulations combined with the metadynamics algorithm, to study antibiotic translocation through $\mathrm{OmpF}$ at a molecular scale. This recently designed algorithm overcomes the time scale problem of classical MD by accelerating some reaction coordinates. It is expected that the initial assumption of the reaction coordinates is a key determinant for the efficiency and accuracy of the simulations. Previous studies using different computational schemes for a similar process only used one reaction coordinate, which is the directionality. Here we go further and see how it is possible to include more informative reaction coordinates, accounting explicitly for: (i) the antibiotic flexibility and (ii) interactions with the channel. As model systems, we select two compounds covering the main classes of antibiotics, ampicillin and moxifloxacine. We decipher the molecular mechanism of translocation of each antibiotic and highlight the important parameters that should be taken into account for improving further simulations. This will benefit the screening and design for antibiotics with better permeation properties.

Response to Reviewers: 


\title{
Investigating reaction pathways in rare events simulations of antibiotics diffusion through protein channels
}

Received: 01.12.2009 / Accepted: 08.03.2010

Eric Hajjar ${ }^{1, \bigotimes}$, Amit Kumar $^{1}$, Paolo Ruggerone ${ }^{1}$, and Matteo Ceccarelli ${ }^{1}$

${ }^{1}$ Department of Physics, University of Cagliari and SLACS-INFM-CNR, I-09042 Monserrato (CA), Italy

${ }^{\square}$ Email: eric.hajjar@dsf.unica.it

\begin{abstract}
In Gram-negative bacteria, outer-membrane protein channels, such as OmpF of Escherichia coli, constitute the entry point of various classes of antibiotics. While antibacterial research and development is declining, bacterial resistance to antibiotics is rising and there is an emergency call for a new way to develop potent antibacterial agents and to bring them to the market faster and at reduced cost. An emerging strategy is to follow a bottom-up approach based on microscopically founded computational based screening, however such strategy needs better-tuned methods. Here we propose to use molecular dynamics (MD) simulations combined with the metadynamics algorithm, to study antibiotic translocation through OmpF at a molecular scale. This recently designed algorithm overcomes the time scale problem of classical MD by accelerating some reaction coordinates. It is expected that the initial assumption of the reaction coordinates is a key determinant for the efficiency and accuracy of the simulations. Previous studies using different computational schemes for a similar process only used one reaction coordinate, which is the directionality. Here we go further and see how it is possible to include more informative reaction coordinates, accounting explicitly for: (i) the antibiotic flexibility and (ii) interactions with the channel. As model systems, we select two compounds covering the main classes of antibiotics, ampicillin and moxifloxacine. We decipher the molecular mechanism of translocation of each antibiotic and highlight the important parameters that should be taken into account for improving further simulations. This will benefit the screening and design for antibiotics with better permeation properties.
\end{abstract}


Keywords Reaction coordinate - Free energy - Accelerated molecular dynamics Antibiotics uptake $\cdot$ Diffusion $\cdot$ Bacterial porins $\cdot$ Antibacterial design 


\section{Introduction}

The permeability to antibiotics - or uptake - is the very first line of defense of Gram-negative bacteria, that are protected by an outer-membrane [1]. In the case of E.coli, the uptake of several classes of $\beta$-lactam antibiotics, a prominent group in our current antibacterial arsenal, is largely controlled by general diffusion protein channels such as Outer Membrane Protein F $(\mathrm{OmpF})$ [1]. Indeed, pathogenic strains of Gram-negative bacteria, that were found to be resistant against quinolones and $\beta$-lactams (two of the main classes of antibiotics) frequently have modulation of the structure or the expression of general diffusion porin OmpF [2].

A key feature in the structure of porins, as seen from the X-ray structure of OmpF [3], is the presence of the loop L3 that folds back into the channel to form a gate, also called constriction region (see Fig. 1A). In addition to such spatial constriction, the zone is also characterized by a strong transversal electric field, generated by negatively charged residues D113, E117 (L3 side) that faces a cluster of positively charged residues R42, R82, and R132 (anti-L3 side) (see Fig. 1).

While antibacterial research and development is on the decline, the resistance is on the rise, and we are thus facing an alarming situation where there is an emergency call for a new way to develop potent antibacterial agents and to bring them to the market faster and at reduced cost. An emerging strategy is to follow a bottom-up approach, from the knowledge of resistant mechanisms to a rational structure-based design and screening of antibiotics. Within this scheme, molecular simulations have the potential to provide an accurate microscopic explanation of what governs antibiotics diffusion (and thus bacterial resistance) and how to screen for better antibiotics. In principle, standard MD simulations would have the required microscopic accuracy to link the structure and dynamics (of the drug and porin) to the rate of permeation. However, standard simulations are limited to hundred of nanoseconds at most and they do not allow the study of the reactive pathway that antibiotics follow during passive diffusion, which is on the order of hundreds of microseconds [4].

To overcome this timescale problem, we propose to use accelerated MD simulation algorithms schemes, or metadynamics, while keeping an "all atom" description of the systems $[5,6]$. The metadynamics algorithm (to accelerate sampling) is based on the following principle: a time-dependent bias is added on a few chosen reaction coordinates (RC). It is important to note that a crucial point of such metadynamic approach is the choice of appropriate RC. 
Unlike other popular techniques (such as steered molecular dynamics [7]), metadynamics allows more than one $\mathrm{RC}$ to be defined and this is important advantage as specifying a single reaction coordinate, can lead to a strong approximation of the sampled process.

Several studies addressed the challenge of such biased molecular dynamics methods to study the difficult problem of molecular diffusion through narrow channels. In their recent paper, Henin et al. [8], highlight the importance of using another reaction coordinates than the usually taken, $\mathrm{Z}$ (distance) coordinate that drives the process under study. Indeed it is believed that including an extra reaction coordinate would allow explicitly to account for reorientations and/or relaxation needed for reaching better accuracy in the sampling of the process studied. In fact, our and others previous studies suggested that the flexibility and orientation of the antibiotic would play a role in its diffusion process through OmpF [5, 9]. However, the qualitative and quantitative consequences of the choice of RC are still poorly understood and there are very few studies illustrating this point. The first natural reaction coordinate that could be used in our metadynamics approach is the position of the antibiotic with respect to the axe of diffusion $\mathrm{Z}$.

Here we go further and include more informative reaction coordinates, accounting not only for the directionality of the transport but also explicitly for: (i) the antibiotic orientation and (ii) specific interactions with the channel. Our approach will allow discussing the two choices of $\mathrm{RC}$ in the translocation with the study of two different antibiotics: the beta-lactam ampicillin and the quinolone moxifloxacine, for which experimental data and simulations are already available $[10,11]$.

Finally, we conclude by discussing the improved strategies, as highlighted by our study. Indeed, the in-depth analysis of these two model systems allows us to discuss for future improvements in the biased methods (importance of explicitly including the relative orientation and the specific interactions) to screen and design for antibiotics in particular, but also for investigating any other complex process of interest. 


\section{Materials and methods}

\section{Starting structures for molecular dynamic simulations}

We followed the same protocol of simulations as described earlier [5], starting from the crystal structure '2OMF' (pdb-code) and residues protonation state as in [12]. We added the required amount of $\mathrm{Cl}-$ and $\mathrm{K}+$ counter ions to neutralize the protein charges. We embedded the system in a hydrophobic environment of detergent molecules (lauryl dimethyl amine oxide, LDAO) and solvated the system with $\sim 8000$ water molecules in an hexagonal box. "Hexagonal periodic boundary conditions were used and the simulation box edges are $68.4 \AA$, $68.4 \AA, 78.1 \AA$. Electrostatic interactions at long distance were evaluated using the soft particle mesh Ewald scheme while a cutoff of $10 \AA$ was used for the Lennard-Jones and short electrostatic energy terms. Multiple Time Step Algorithm (MTS Respa) was used with the SHAKE algorithm to keep bond lengths involving hydrogens fixed. The simulations were done at $300 \mathrm{~K}$ with Nose thermostat to control temperature.

We used the Amber potential and TIP3P for water [13]. The parameters of antibiotics were developed following the Amber protocol [14]: (i) the three dimensional chemical structure of the antibiotic was drawn, using the software package HyperChem; (ii) geometry optimization was performed using the Hartree-Fock (HF) basis set HF-6-31G* with the Gaussian03 package [15]; (iii) the molecular electrostatic potential was generated at HF/6-31G* level; (iv) the atomic charges were fitted to molecular electrostatic potential with aid of a module RESP in Amber8 program package, adding the restraint for equivalent atoms [14]. The structure obtained after full optimization is considered the starting geometry of the molecule onto which we assign all the force-field parameters. The atom types and parameters were derived using either the program antechamber (Amber-module), when possible, or were assigned manually on the basis of our optimized geometry. We used the program ORAC and the Amber force field [13] for system setup and simulation [16]. All simulated systems were validated for convergence and stabilization of energy, temperature and root mean square deviation with respect to the starting structure. 


\section{Metadynamics algorithm}

The metadynamics algorithm employs a bias to accelerate the evolution of some collective variables, defined as the relevant reaction coordinates for the process under investigation. The bias consists of a history dependent potential, which is constructed as a sum of repulsive potential centered along the trajectory of the collective variables. These additional energy terms avoid revisiting the same conformations or at least add a penalty term to the previously visited conformations. In the present simulations, a Gaussian potential is added every $4 \mathrm{ps}$ with a height is of $1.0 \mathrm{~kJ} \mathrm{~mol}^{-1}$. The Gaussian width is set to $0.2 \AA, 5.0$ degrees and 0.5 , respectively for the distance $Z$, the angle $\Theta$ and the number of hydrogen bonds. These parameters were chosen to allow a better resolution in the sampling of the free energy and a low error (around $2 \mathrm{k}_{\mathrm{B}} \mathrm{T}$ ).

The most important point in the metadynamics is to select the proper reaction coordinates. These must be variables that are of interest but difficult to sample with a standard scheme, since the local minima in the space spanned by these variables are separated by barriers that cannot be overcome in the simulation time available. We also wanted to compare the effect of choosing different collective variables in this case, and thus we chose the three different collective variables:

(i) Distance (Z), defined as: $Z=Z_{G E O}($ ant $)-Z_{G E O}($ solute $)$,

where $\mathrm{Z}_{\mathrm{GEO}}$ is calculated as the average of $\mathrm{Z}$ coordinates of all the heavy atoms of the system, respectively antibiotics and (porin+detergent)

(ii) Angle $(\Theta)$, defined as: $\operatorname{Cos}(\theta)=e_{Z} \cdot v_{\text {ant }}$,

with $\mathrm{e}_{\mathrm{Z}}$ being the eigenvector of inertia tensor (calculated on the porin $\mathrm{C} \alpha$ ) that is closest to the axe of diffusion of the porin, $v_{a n t}$ being the vector of the long axis of the antibiotic, the closest to the highest component of the dipole moment.

(iii) Number of hydrogen bonds, defined as a continuous function from the list of all

defined donors and acceptors of the system: $N_{H B}=\sum_{i=1}^{n H B} \frac{1-\left(r_{i} / r_{0}\right)^{6}}{1-\left(r_{i} / r_{0}\right)^{12}}$, 
where $\mathrm{nHB}$ is the total number of possible hydrogen bonds calculated at time $\mathrm{t} ; \mathrm{r}_{0}$ is the reference distance between the two heavy atoms of the bonds taken as $2.5 \AA$.

The metadynamics algorithm enables the reconstruction of the free energy in the subspace of the collective variables by integrating the history dependent terms [6]. Due to the complexity of the process studied, we calculated the free energy after obtaining the first translocation path, which is considered to be the most probable path because it passes through the lowest saddle point, as done before for the unthreading of a molecule [17]. In fact, once the antibiotic crosses the constriction region, we expected a diffusive regime, with no significant affinity sites. The error bars associated with the energy calculations were assessed as previously done [17] and are of $1 \mathrm{kcal} \mathrm{mol}^{-1}$ at most.

\section{Microscopic analysis methodology}

To decipher the molecular details of the translocation mechanism additional equilibrium MD simulations (1 ns length) were started from each visited minima along the diffusion path. In depth analysis using VMD and in-house scripts, was performed to characterize the following key structural features:

(i) The atomic root mean square fluctuations (rmsf) were calculated for each heavy atoms of the backbone of the antibiotic with respect to its average position during the MD simulations;

(ii) Existence of hydrogen bonds $(\mathrm{Hb})$ and hydrophobic contacts $(\mathrm{Hc})$ between atoms of the antibiotics and of OmpF. Hbs are counted using VMD scripts according to the following threshold parameters: a distance of at most $3 \AA$ and donor-hydrogen-acceptor angle of at least 130 degrees. Hcs are counted when non-polar atoms are separated by at most $3 \AA$.

Further, we report the molecular properties in terms of the amount of hydrophilic/hydrophobic surface, as calculated from the PLATINIUM server [18] . 


\section{Results and discussion}

\section{Metadynamics simulations allow studying the translocation process}

Both ampicillin and moxifloxacine have a permanent dipole, being its larger component along their long axe (Fig. 1 B-C). As during permeation, antibiotics have to penetrate the constriction region that is rich of charged amino acids, it is important to accurately define the way of entry and the interactions with the channel. To do so, we included explicitly these degrees of freedom as reaction coordinate along the metadynamics runs.

The Fig. 2 displays the free energy surfaces (FES) of the translocation of the ampicillin betalactam antibiotic (Amp) through the OmpF channel for two different choices of the reaction coordinates: (A) with the combination of the distance $\mathrm{Z}$ and the number of hydrogen bonds $\mathrm{Hb}$; and (B) with the combination of the distance $\mathrm{Z}$ and the angle $\Theta$. Interestingly, the free energy barrier required for ampicillin to translocates is of the same order in the two simulations: $11 \mathrm{kcal} \mathrm{mol}^{-1}$ and $9 \mathrm{kcal} \mathrm{mol}^{-1}$ for the simulation $\mathrm{A}$ and $\mathrm{B}$ respectively (the values correspond to the energy barrier needed to translocates starting from the deepest minima above). In both cases, deep energy minima are visited, which can be related to well defined affinity sites of ampicillin inside the channel. In particular, we observe the location of a deep energy minimum exactly at the constriction region (Mini-CR at Z 0, see Fig. 2). To address the similarity (reproducibility) of the process sampled with different RC, we further calculated the missing $\mathrm{RC}$ of each simulation and plot it in the corresponding FES. Thus, the $\mathrm{Hb}$ coordination number, as calculated in the course of the simulation B, were superimposed as blue points in Fig. 2A; similarly, the $\Theta$ angle, as calculated in the course of the simulation A, were superimposed as blue points in Fig. 2B. Interestingly, the number of hydrogen bonds needed (about $10 \mathrm{Hbs}$ ) to cross the constriction region and translocate is similar in both simulations that were "constrained" (A) or "free" (B) of this RC. Similarly, both simulations sample the same populations of states of the angle $\Theta$ (the value of $\Theta$ when ampicillin crosses the constriction region and translocates is around 150 degrees in both simulations A and B) defining the deep minima at the constriction region. The fact that both the angle and the hydrogen bond coordinate are reproducing a very similar path, with a deep energy minimum located centrally at the CR, comforts us in the choice of these RC. For the study of ampicillin's translocation, both RC are appropriate. 
Next, we performed metadynamics simulations using the same two sets of RC for the quinolone antibiotic: moxifloxacine. As seen in the FES displayed in Fig. 3, there are some important differences between the two sets of simulations for the translocation of moxiflocacine. First, the free energy barrier required for moxiflocacine to translocate is much larger in the simulation A, $21 \mathrm{kcal} \mathrm{mol}^{-1}$, compared to the simulation $\mathrm{B}, 16 \mathrm{kcal} \mathrm{mol}^{-1}$. The localization of the energy minima, defining the affinity sites of the antibiotic inside the channel, is also very different. In the simulation A we note the absence of a deep energy minimum at the constriction region, instead it is localized slightly above, at $Z \sim 4 \AA$ (MiniAbove, Fig. 3A). In the case of the simulation B we find two energy minima localized above but also in particular one energy minimum centrally localized, at the constriction region, at $\mathrm{Z}_{\text { }}$ $0 \AA$ (Mini-CR, Fig. 3B).

The important differences between these simulations using different $\mathrm{RC}$ are seen from the superimposition in the FES obtained from the simulation with a RC of the calculated values of the missing RC. As seen in the blue points superimposed in Fig. 3A, the hydrogen bonds calculated from the simulation B do not follow the same path as the Hb sampled from simulation $\mathrm{A}$. Instead, the number of $\mathrm{Hb}$ calculated from the simulation $\mathrm{B}$ is both more constant and lower than the number of $\mathrm{Hb}$ sampled along simulation $\mathrm{A}(\sim 4 \mathrm{Hb}$ from simulation $\mathrm{B}$ while there are $8 \mathrm{Hb}$ when the antibiotic crosses the constriction region in simulation A). Similarly, as seen in the Fig. 3B, the superimposed $\Theta$ angles calculated from simulation A do not really follow the path of the $\Theta$ angles sampled in simulation B. Instead, the $\Theta$ angles calculated from simulation A reveal a much more stretched and limited exploration path (the $\Theta$ angles only take values from 40 to 120 degrees in the case of the simulation A, whereas they can cover fully the range from 0 to 180 in the case of simulation B), as a consequence some local minima (above the constriction region) are not sampled in the path followed by the simulation A (Fig. 3).

To elucidate the microscopic details of the different translocation path and conclude on the importance of the choice of the RC we further performed in-depth analysis based on equilibrium simulations. 


\section{Equilibrium MD simulations allow studying the structural and dynamics properties}

In order to characterize the structural and dynamics properties that govern the antibiotics diffusion process we then performed equilibrium MD simulations (of 1ns length) starting from the relevant, preferential minima identified by the FES. We focus only on the minima where the antibiotics are bound at the constriction region of the channel (Mini-CR) as if such interaction was the rate-limiting step of the process. In the case of ampicillin, we find that very similar structures are sampled along the two Mini-CR from both simulations with the $\mathrm{Hb}$ and $\Theta$ RC, we will further only describe the Mini-CR of the simulation B (with the $\Theta \mathrm{RC}$ ). As seen from the snapshot in Fig. 4A, to enter the affinity site at the constriction region (Mini$\mathrm{CR}$ ) and further translocates, ampicillin is oriented with its polar carboxyl group pointing down and makes favorable interactions with the residues of the porin constriction zone. We present in Fig. 4B the lifetime of the hydrogen bonds and hydrophobic contact $(\mathrm{Hc})$ interactions between ampicillin and OmpF atoms sampled along Mini-CR (as explained in the Method section). We observe that ampicillin is able to make a large number of durable polar $\mathrm{Hbs}$, in particular both the one between its N-terminal positive group (N1) and D113 and the one between its C-terminal carboxylic group (O2) and R132 exceed $70 \%$ of the equilibrium simulation time. Instead, only one significant Hc ( 20\% of the simulation time) is made between the CT2 carbon of ampicillin and M114 (Fig. 4B).

A similar detailed structural analysis was performed when moxifloxacine is bound in Mini$\mathrm{CR}$, in the simulation $\mathrm{B}$ (with the $\Theta \mathrm{RC}$ ), and we find that in this case, the antibiotic is oriented with its polar carboxyl group pointing up (Fig. 5A). Instead, it is with its hydrophobic group that moxifloxacine is seen to penetrate the constriction region (Fig. 5A). The interaction network analysis indeed reveals a predominance of Hcontacts between the antibiotic and the many hydrophobic residues on the porin side such as F118, L20, M114, P116 (Fig. 5B). The only significant Hb identified involves Y124, E117 and to a lesser extend $\mathrm{R} 82$.

Differences in the structural, dynamical or physico-chemical properties of the two antibiotics could explain the differences observed in our study - that is, the translocation of ampicillin is described equivalently by both the $\mathrm{Hb}$ and the $\Theta \mathrm{RC}$ while the translocation process of moxifloxacine differs if choosing the $\mathrm{Hb}$ or the $\Theta$ RC. Table 1 reports diverse physicochemical properties of the two antibiotics, such as the flexibility and solvation pattern, 
obtained from the optimized 3D structures or from simulations where they are placed in a box of water. We observe that, although both antibiotics have the same molecular surface size, ampicillin is found to be much more polar while moxifloxacine is much more hydrophobic (see computed surface properties in Table 1). This is in agreement with the antibiotics nature of interactions with of the antibiotics with OmpF, as . Furthermore, based on RMSF Interestingly, we also find that the ampicillin structure is twice more flexible than the one moxifloxacine (see calculated rmsf in Table 1).

\section{Conclusions}

Here we used all-atom metadynamics simulations with different choice of the RC and followed the translocation through OmpF of two commonly used antibiotics. The high resolution in time and space that metadynamics simulations provide has the potential to be used to characterize the molecular basis of translocation of antibiotics, and further, such strategy might be used to help select/design antibiotics with better permeation properties and thus combat bacterial resistance. To improve the accuracy and efficiency of future simulations efforts, we must keep on developing the algorithms at hands. Here we address the consequences of chosen different $\mathrm{RC}$, which is the crucial point of the metadynamics techniques. Due to the cylindrical shape of OmpF, a tentative choice of RC is the position of the antibiotic along the main axe of diffusion. Our results on moxifloxacin and ampicillin support our hypothesis that the $\Theta$ angle defines an optimal RC, as it is an internal variable not subject to change as OmpF diffuses during simulation.

Altogether, from our simulations, we observe that the bottleneck for the antibiotics translocation is to overcome the constriction region, where the antibiotic has to optimally interact with the channel, its dipole has to match the strong transversal electrostatic field and its surface has and adapt to fit to a given size.

In the simulation where the $\mathrm{Hb}$ was taken as a $\mathrm{RC}$, we found that ampicillin can make and break $\mathrm{Hb}$ easily and it reaches up to $10 \mathrm{Hb}$ but in the case of moxifloxacine this number is constant and as low as 4, thus we believe that overcoming the constriction is more difficult in the case of a less polar or more hydrophobic antibiotic. The molecular details of the simulations allow assessing how the choice of the RC affects the FES and the molecular mechanism for antibiotic translocation. 
In the case of ampicillin, although metadynamics is applied with different $\mathrm{RC}$, we find a similar antibiotic translocation pathway. In both cases, a unique energy minimum is found for ampicillin at the constriction region, which is well defined with a strong interaction network. In this preferential minimum, the (two charged groups) dipole of the antibiotic matches perfectly with the one of the porin, known as the transversal electric field, with negative residues clustered on the L3 side and positive residues lining on the anti-L3 side (see Fig. 1).

Interestingly, a different molecular path is found by moxifloxacine with respect to ampicillin: it translocates with its hydrophobic group pointing down and we note a predominance of Hcontacts interactions that play an important contribution as a driving force. Such possible contribution from hydrophobicity was already raised in the pioneer work of Nikaido [19]. Indeed, the newer generations of antibiotics that are more hydrophilic have enhanced antibacterial activity, indicating that hydrophilicity would enhance their affinity to OmpF. Thus, in such cases, we suggest to include directly the hydrophobic interactions number as a $\mathrm{RC}$ in the course of metadynamics simulations. Further analysis allowed extracting important structural and dynamical properties of the antibiotics, such as solvation and flexibility, and the same could be done with the porin target.

It is noteworthy that the two different sets of reaction coordinates used in this manuscript reproduce well the previously obtained experimental observations [10, 11]. Indeed, both resulting simulations reveal the same "macroscopic event" that is the passage/diffusion of the antibiotic and both find a preferential affinity site for the antibiotic inside the channel. However, using different reaction coordinates leads to different simulation time and efficiency. In this particular manuscript we aim at improving the efficiency of the molecular simulations and we show how the "right choice of coordinate" could be primordial for doing so. The importance of having an efficient and reliable computational model for antibiotic translocation is also highlighted by our recent findings that pointed out the limitations, in time and space resolutions, of kinetic experiments [20].

To conclude we believe that to gain in efficiency and accuracy, the antibiotic translocation could be simulated with many RC coupled together: allowing to account explicitly for solvation, flexibility and different possible specific interactions/properties of importance in the system of study. This could be done by using a scheme such as the "bias exchange metadynamics“ recently proposed [21]. 
Another possibility is to use a bias-free method [22], but then a way must be found to sample the trajectory corresponding to the studied process (for example, by using temperature as a bias as in TAMD simulations [23]). Such explorative strategies would in fact reveal which RC is relevant for the process and system studied. Better-tuned simulation methodology will be also useful to investigate many diverse complex biological processes, such as folding or protein-protein interactions.

\section{Acknowledgments}

We thank Attilio Vargiu and Enrico Spiga for fruitful discussions. This study was supported by EU-grant MRTN-CT-2005-019335 (Translocation) and by the computer center and consortiums: Cybersar, CASPUR and CINECA through CPU-hours. 


\section{References}

1. Nikaido H (2003) Molecular basis of bacterial outer membrane permeability revisited. Microbiol Mol Biol Rev 67:593-656

2. Chevalier J, Mallea M, Pages JM (2000) Comparative aspects of the diffusion of norfloxacin, cefepime and spermine through the F porin channel of Enterobacter cloacae. Biochem J 348:223-227

3. Cowan SW, Schirmer T, Rummel G, Steiert M, Ghosh R, Pauptit RA, Jansonius JN, Rosenbusch JP (1992) Crystal structures explain functional properties of two E. coli porins. Nature 358:727-733

4. Nestorovich EM, Danelon C, Winterhalter M, Bezrukov SM (2002) Designed to penetrate: time-resolved interaction of single antibiotic molecules with bacterial pores. Proc Natl Acad Sci USA 99:9789-9794

5. Ceccarelli M, Danelon C, Laio A, Parrinello M (2004) Microscopic mechanism of antibiotics translocation through a porin. Biophys J 87:58-64

6. Laio A, Parrinello M (2002) Escaping free-energy minima. Proc Natl Acad Sci USA 99:12562-12566

7. Robertson KM, Tieleman DP (2002) Orientation and interactions of dipolar molecules during transport through OmpF porin. FEBS Lett 528:53-57

8. Henin J, Tajkhorshid E, Schulten K, Chipot C (2008) Diffusion of glycerol through Escherichia coli aquaglyceroporin GlpF. Biophys J 94:832-839

9. Vidal S, Bredin J, Pages JM, Barbe J (2005) Beta-lactam screening by specific residues of the OmpF eyelet. J Med Chem 48:1395-1400

10. Mach T, Neves P, Spiga E, Weingart H, Winterhalter M, Ruggerone P, Ceccarelli M, Gameiro P (2008) Facilitated permeation of antibiotics across membrane channels-interaction of the quinolone moxifloxacin with the OmpF channel. J Am Chem Soc 130:13301-13309

11. Danelon C, Nestorovich EM, Winterhalter M, Ceccarelli M, Bezrukov SM (2006) Interaction of zwitterionic penicillins with the OmpF channel facilitates their translocation. Biophys J 90: 1617-1627

12. Im W, Roux B (2002) Ions and counterions in a biological channel: a molecular dynamics simulation of OmpF porin from Escherichia coli in an explicit membrane with $1 \mathrm{M} \mathrm{KCl}$ aqueous salt solution. J Mol Biol 319:1177

13. Cornell WD et al (1995) J Am Chem Soc 117:5179 
14. Wang J, Wolf RM, Caldwell JW, Kollman PA, Case DA (2004) Development and testing of a general amber force field. J Comput Chem 25:1157-1174

15. Frisch MJ et al (2004) Gaussian 03, Revision C02. Gaussian Inc, Wallingford CT

16. Procacci P, Darden TA, Paci E, Marchi M, (1997) ORAC: a molecular dynamics program to simulate complex molecular systems with realistic electrostatic interactions. J Comput Chem 18:1848-1862

17. Laio A, Rodriguez-Fortea A, Gervasio FL, Ceccarelli M, Parrinello M (2005) Assessing the accuracy of metadynamics. J Phys Chem B 109:6714-6721

18. Pyrkov TV, Chugunov AO, Krylov NA, Nolde DE, Efremov RG (2009) PLATINUM: a web tool for analysis of hydrophobic/hydrophilic organization of biomolecular complexes. Bioinformatics 25:1201-1202

19. Yoshimura F, Nikaido H (1985) Diffusion of beta-lactam antibiotics through the porin channels of Escherichia coli K-12. Antimicrob Agents Chemother 27:84-92

20. Hajjar E, Mahendran KR, Kumar A, Bessonov A, Petrescu M, Weingart H, Ruggerone P, Winterhalter M, Ceccarelli M (2010) Bridging Timescales and Length Scales: From Macroscopic Flux to the Molecular Mechanism of Antibiotic Diffusion through Porins. Biophys J. doi:10.1016/j.bpj.2009.10.045

21. Piana S, Laio A (2007) A bias-exchange approach to protein folding. J Phys Chem B 111:4553-4559

22. Branduardi D, Gervasio FL, Parrinello M (2007) From A to B in free energy space. J Chem Phys 126:54103

23. Weinan E, Vanden-Eijnden E (2010) Transition-Path Theory and Path-Finding Algorithms for the Study of Rare Events, Annu Rev Phys Chem. doi:10.1146/annurev.physchem.040808.090412 


\section{Tables}

Table 1 Structural details of the antibiotic obtained from the equilibrium MD simulations. (see Materials and Methods)

\begin{tabular}{|c|c|c|c|}
\hline \multirow{2}{*}{$\begin{array}{l}\text { System Analysis } \\
\text { (in box of waters) }\end{array}$} & \multirow{2}{*}{$\begin{array}{c}\text { Averaged } \\
\text { Atomic } \\
\text { Fluctuations } \\
\text { (rmsf in } \AA \text { ) }\end{array}$} & \multicolumn{2}{|c|}{ Surface Properties [18] } \\
\hline & & Hydrophillic & Hydrophobic \\
\hline AMP & 0.99 & 221.45 & 83.92 \\
\hline MOX & 0.48 & 156.52 & 175.63 \\
\hline
\end{tabular}




\section{Figure captions}

Fig. 1 A) Structural details of OmpF. The backbone of OmpF is displayed in cyan cartoons to highlight its secondary structure. The charged residues at the constriction region (D113, E117, D121 on the L3 side and R42, R82, R132 on the anti-L3 side) are colored by residue type (positively charged in blue, negatively charged in red). The loop L3 is colored in orange. 3D structures of the optimized geometry of ampicillin (B) and (C) moxifloxacin. The molecules are colored by atom types: blue for nitrogen, red for oxygen, cyan for carbon.

Fig. 2 Complete free energy surfaces for ampicillin translocating through OmpF, associated with the reaction pathway along the subspace of the two variables: taken as (i) the distance $Z$ ( $Z=0$ corresponds to the center of the constriction zone, also indicated by the grey shading) and the number of $\mathrm{Hb}(\mathbf{A})$; and taken as (B) the distance $\mathrm{Z}$ and the angle $\Theta$. Each isoline correspond to a different color gradient and to $1 \mathrm{kcal} \mathrm{mol}^{-1}$. The blue points superimposed on the FES correspond to the superimposed values of the RC calculated from the other simulation.

Fig. 3 Complete free energy surfaces for moxifloxacine translocating through OmpF (see legends in Fig. 2)

Fig. 4 A) Molecular detail of ampicillin at the preferred minimum, at the constriction region, Mini-CR, showing the interacting residues of $\mathrm{OmpF}$ (colored by residue types, the ones involved in Hbs are displayed as sticks and those involved in Hcontacts are displayed by molecular surface) (see Fig. 1 for coloring code). B) Interaction map for the equilibrium $\mathrm{MD}$ of ampicillin in the preferential minimum, at the constriction region (Mini-CR). In the $\mathrm{x}$-axis is represented each atom of the antibiotic, in $y$-axis is calculated the lifetime (probability of existence along simulation) of each interaction between atoms of the antibiotic and atoms of OmpF. In black bars are represented the hydrogen bonds $(\mathrm{Hb})$ and in grey bars the hydrophobic contacts (Hcontacts), calculated as explained in Material and Methods.

Fig. 5 Molecular details (A) and interaction map (B) of moxifloxacine at the preferential minimum, Mini-CR (see legends in Fig. 4) 

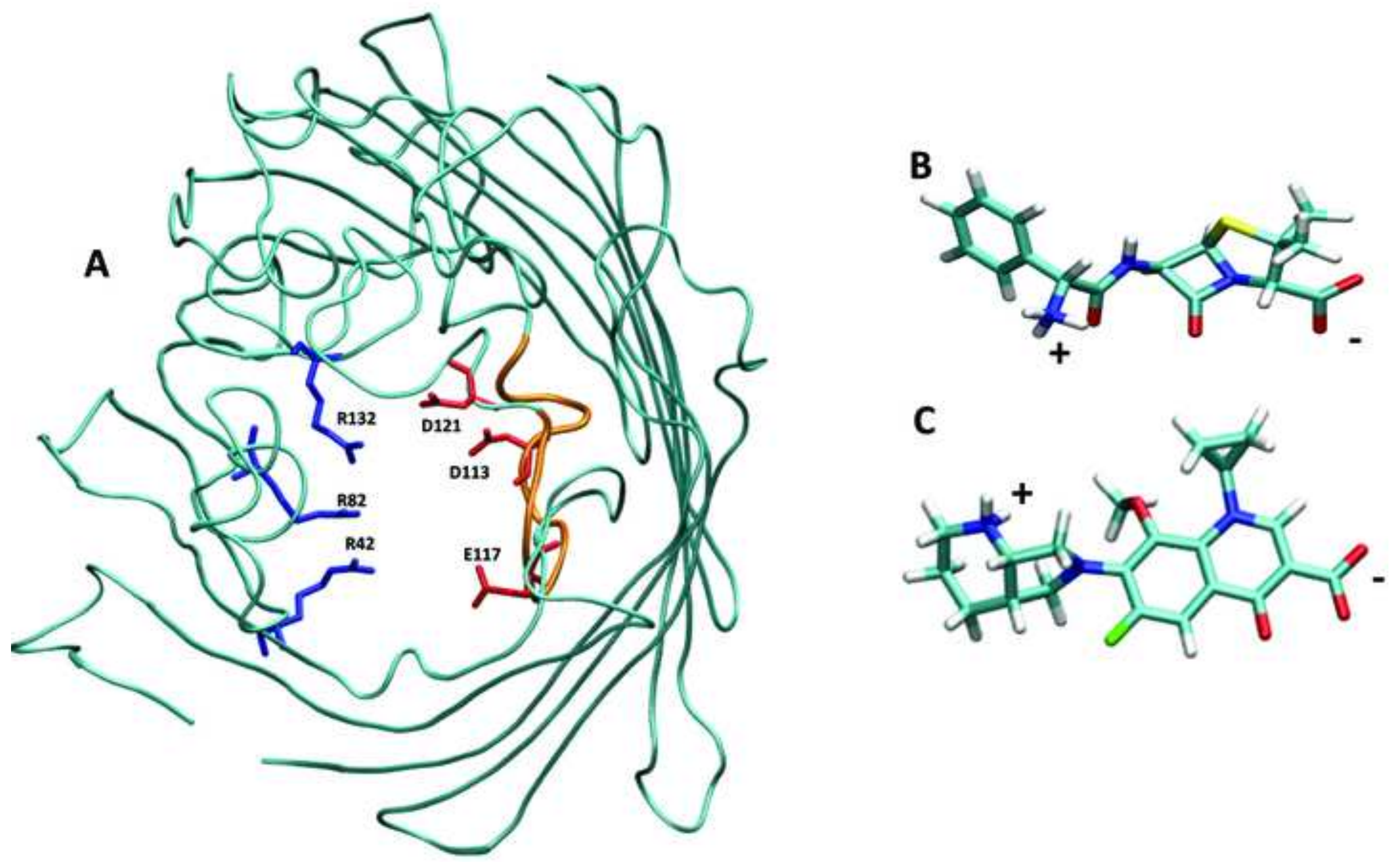
Click here to download high resolution image

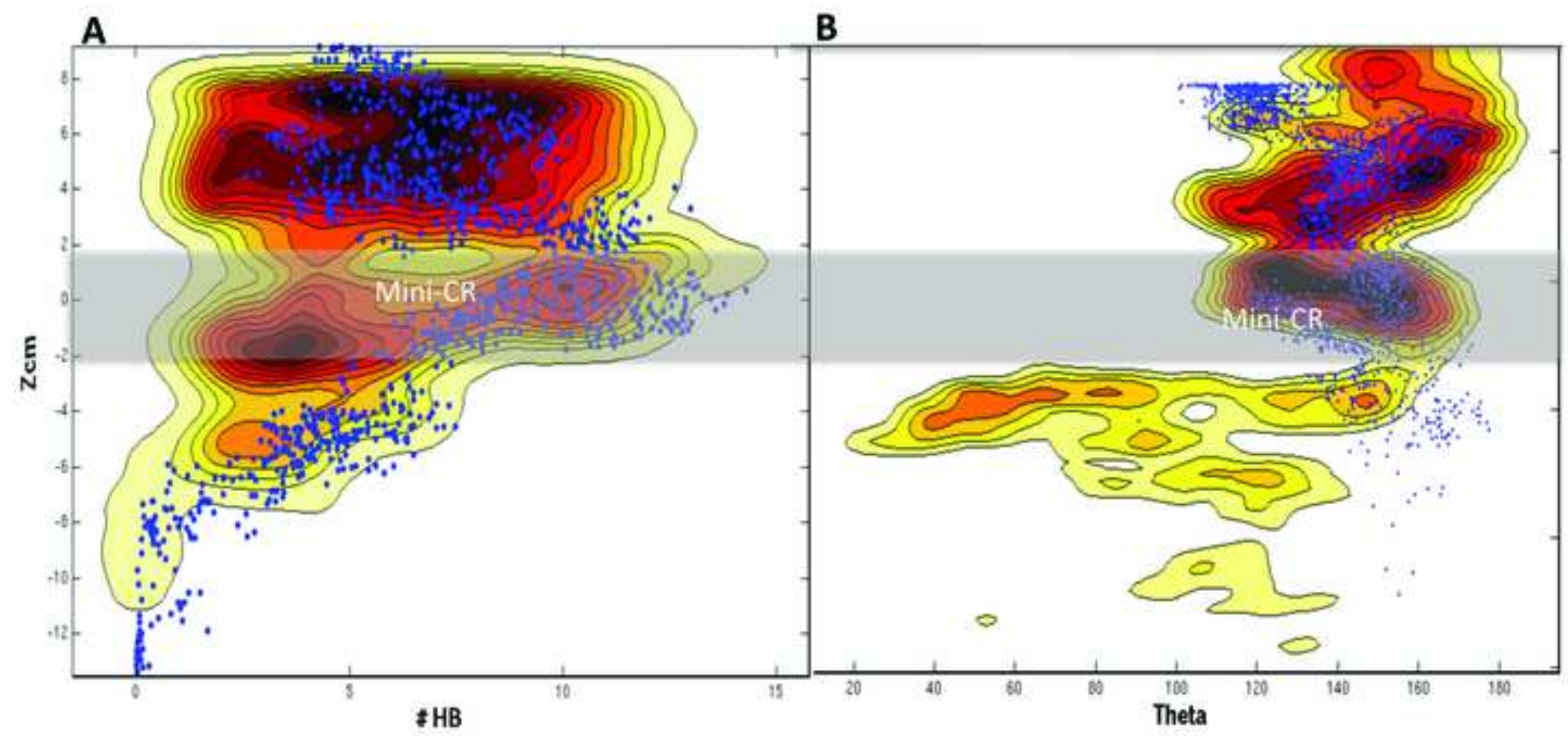


Click here to download high resolution image

A

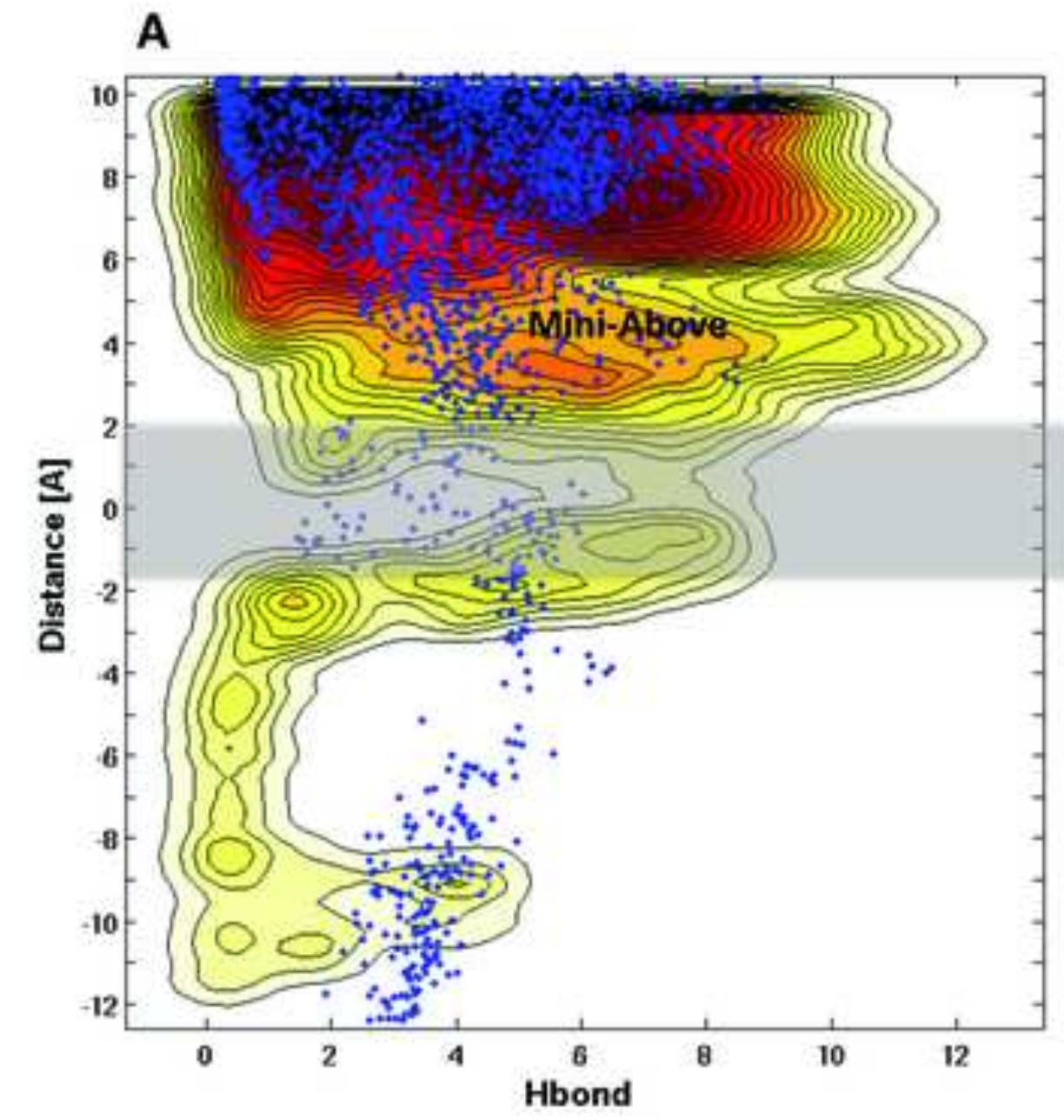

B

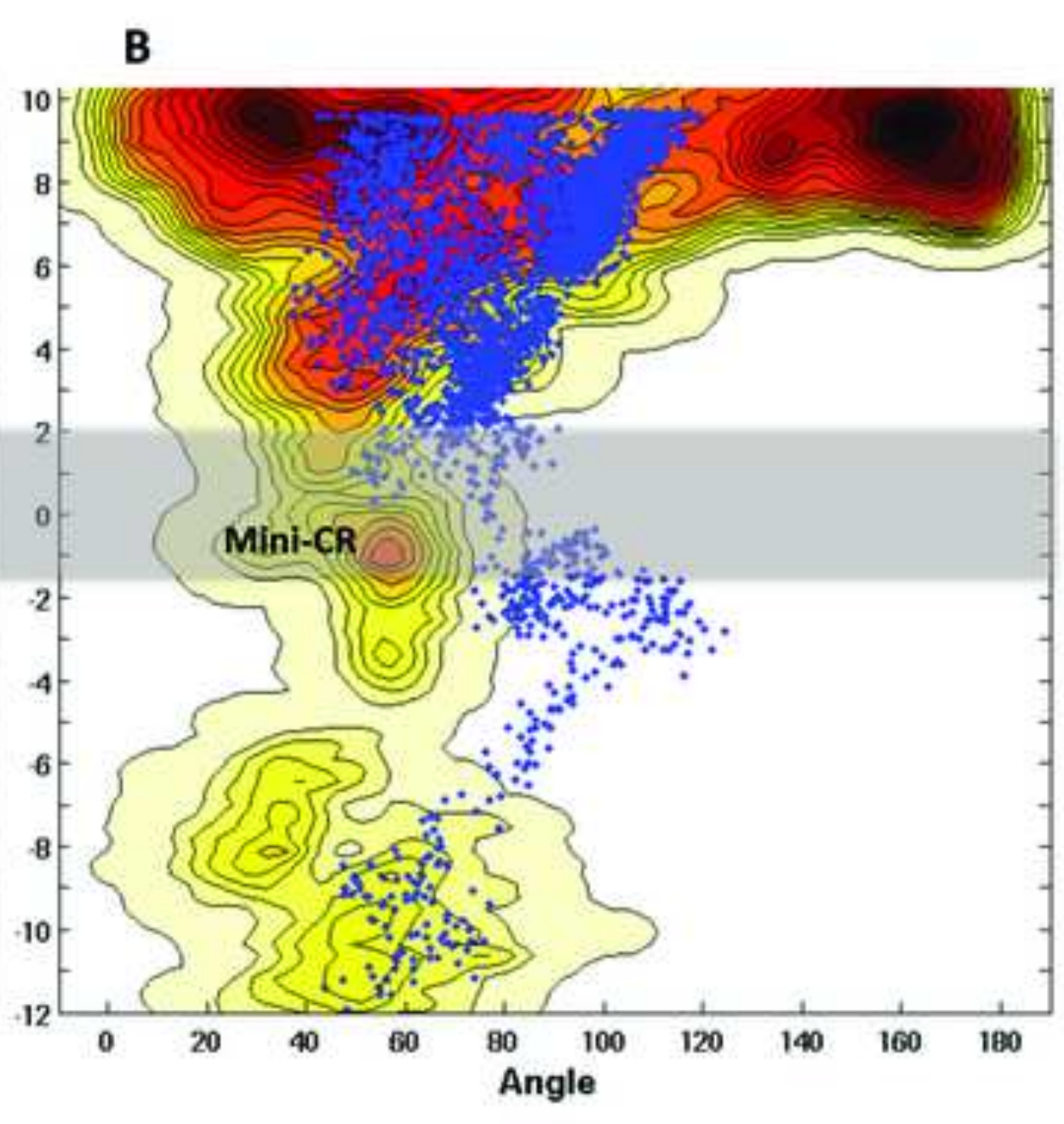



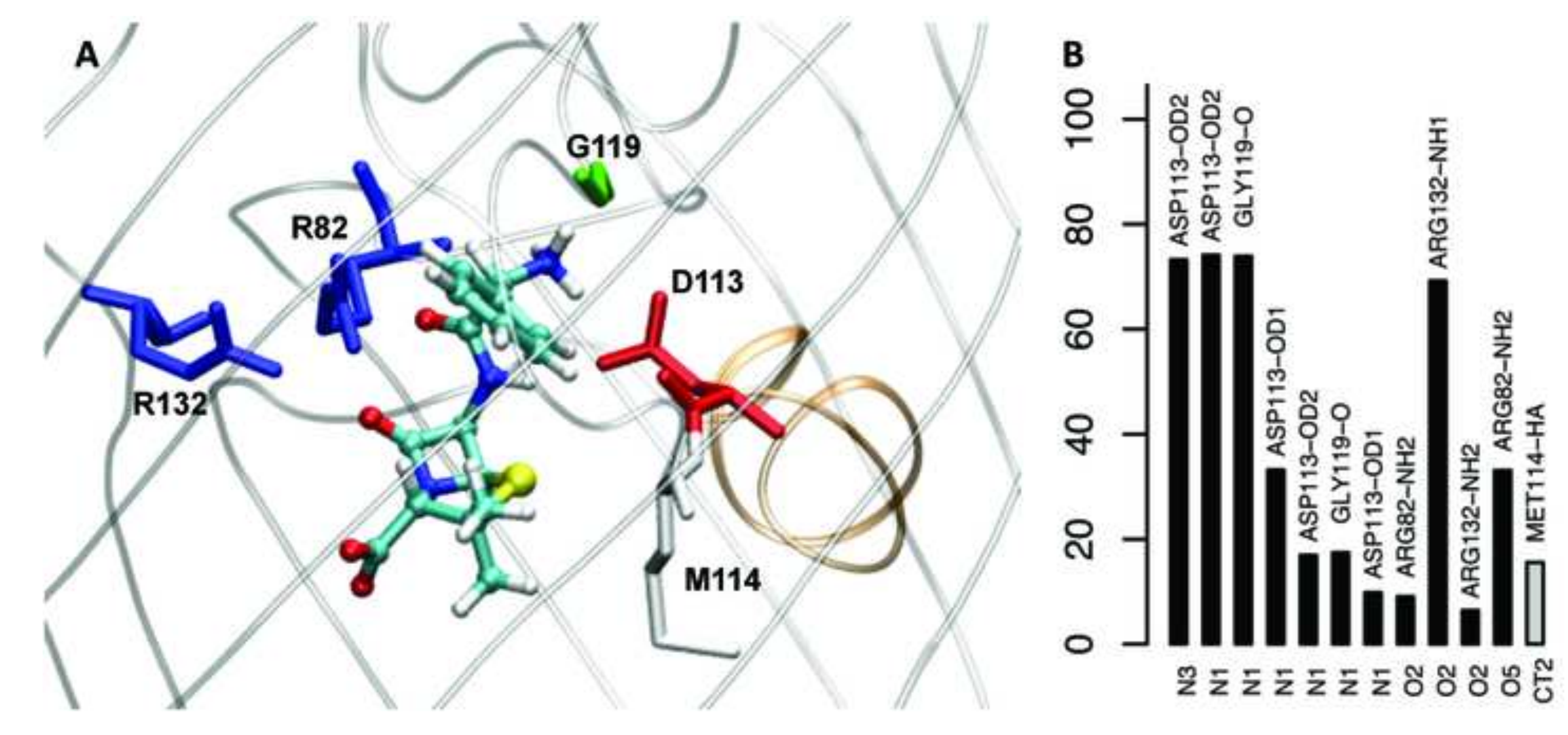

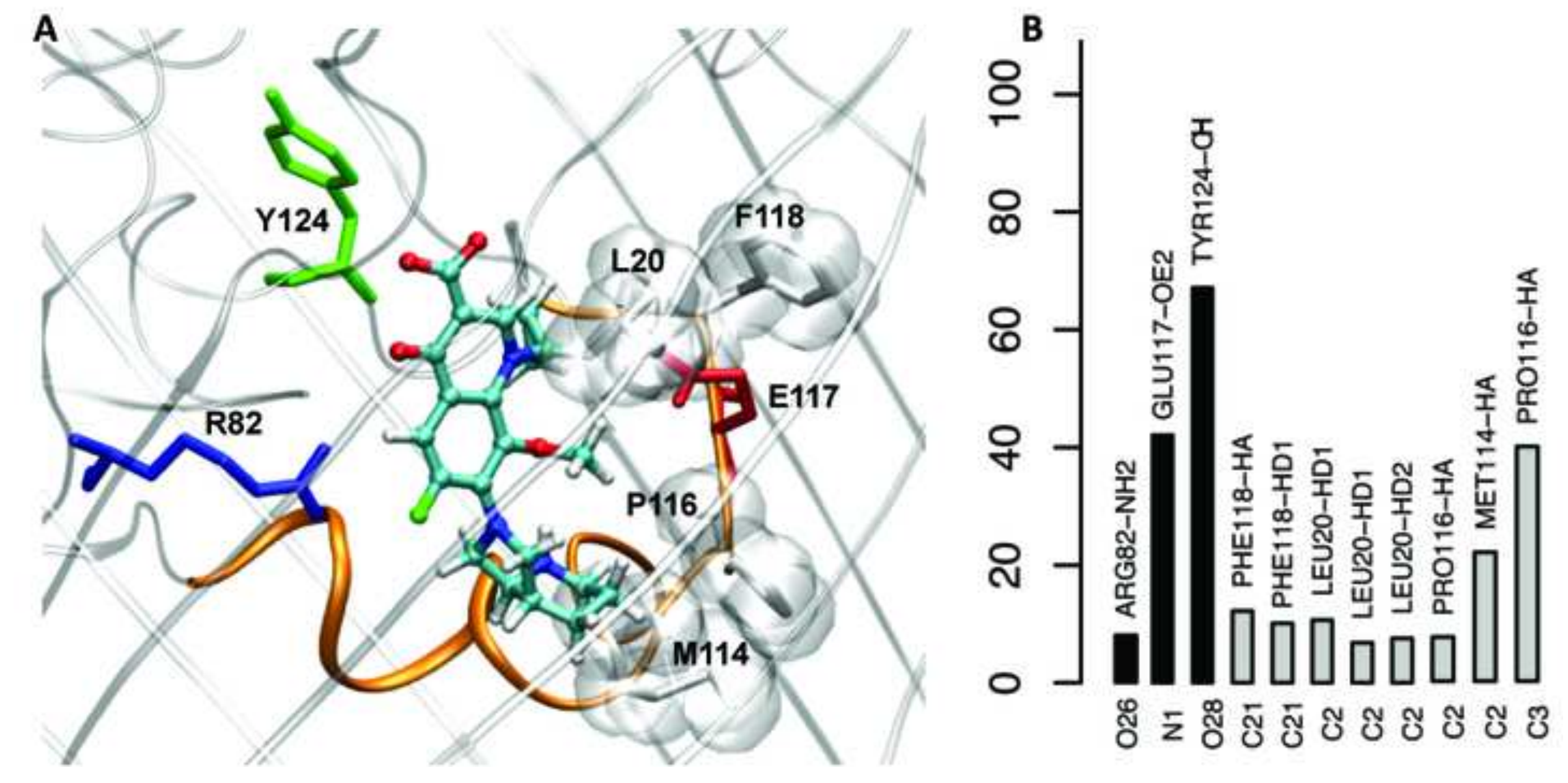